\title{
Analysis of the Development Situation of Chinese Socialization E-commerce
}

\author{
Xiaoyi Zhu,Bin Huang \\ School of Media Studies \& Humanities ,Zhejiang University City College ,Hangzhou, China \\ E-mail: sober4125@gmail.com
}

\begin{abstract}
At present, with the continuous emergence of social network sites of different types in China, the socialization of ecommerce has emerged as a new trend. However, development of this commerce sector has been relatively weak thus far. This thesis aims to discuss current development patterns of Chinese socialization e-commerce and problems that may occur during the development process, and will also propose possible solutions.
\end{abstract}

Keywords-development situation; China; socialization; ecommerce

*Xiaoyi Zhu is the first author; Bin Huang is the correspondence author.

\section{INTRODUCTION}

Socialization e-commerce is a new derivation pattern of e-commerce. It combines the social nature of social networking websites that allow people to see and access goods and services enjoyed by their contacts with ecommerce opportunities. China is new to the field and in the early stages of development for effective social e-commerce strategies.

\section{THREE MAIN DEVELOPMENT PATTERNS AND FEATURES OF CURRENT CHINESE SOCIALIZATION E- COMMERCE}

Wherever Times is specified, Times Roman or Times New Roman may be used. If neither is available on your word processor, please use the font closest in appearance to Times. Avoid using bit-mapped fonts if possible. True-Type 1 or Open Type fonts are preferred. Please embed symbol fonts, as well, for math, etc.

\section{A. Socialization e-commerce platform based on acquaintances}

This pattern is typically represented by "Renren shopping," introduced by Renren Corporation, a social networking site similar to Facebook. Users can quickly and easily discover dresses, accessories and cosmetics they like according to their own preferences and habits through daily online interaction with friends. Sharing and recommendations achieve high reliability in this platform. The marketing is already built in, as recommendations come from a potential buyer's own circle of friends.

1) Real-name system social contact feature increases shopping trust

The social media age makes everyone's social network clearer. Websites like Renren mostly refer to acquaintances, relatives, friends and classmates. Trust is built into these online communities, and the endorsement of a friend or family member is quite valuable to online shoppers. The social networks also indicate users'/shoppers' ages and genders, allowing e-commerce sites to tailor their advertising to a specific market or type of user.

This data graph, recently issued by ODM GROUP, concentrates on providing digital solutions (please refer to figure 1). The graph indicates that $90 \%$ of consumers trust the opinions of their acquaintances; the effect on consumers of the social circle in a real world is greater than experts, advertisements and celebrities; consumers in the real social circle and online social circle enjoy a higher level of trust.

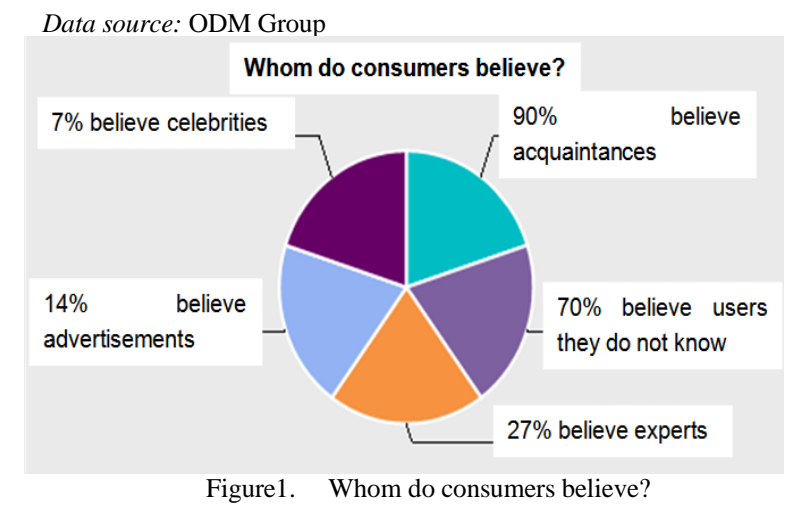

The investigation of Nielsen for Renren in 2011 even pointed out: $57 \%$ of Renren users recommend products to others; $40 \%$ would accept recommendations from friends on social networks and buy products; $60 \%$ would trust brands recommended by friends more than those that are not; $16 \%$ are willing to buy more trusted brands based on recommendations.

\section{2) Information value is higher}

Users will issue honest evaluation in real social networks, which avoids false or propagandized reviews.

\section{B. Shopping-guide Communities based on Interest Connection}

This is typically represented by Mushroom Street and Beauty Discussion. Users are linked together out of some specific topic or common interest in a certain product. The shopping guide helps them discover and choose products.

1) Most are female users

This kind of community is mostly related to female users who purchase products related to fashion, beauty and home furnishings. It can be inferred from user distribution that 
Chinese female netizens are active on shopping-guide social commerce platforms.

2) Pictures of falls' presence attract users more

Falls, also known as falls' layout, is a kind of popular website page layout based on interest connection in shopping-guide communities with a visual organization of a jagged multi-column layout. Using a downward scolling function, this layout will continuously upload data blocks and add them to current ending.

Like most girls who go shopping in actual stores, this allows them to browse from the comfort of wherever they are. If pagination is not dealt with for products in short space, users will be immersed in contents, thus products achieve purpose and users' thoughts would not be interrupted out of pagination.

3) Efficient information filter

The shopping-guide platform solves the problem of information filtering for mainstream users who daunted when searching from products on TaoBao which has more than 800 million product listings. People are linked together due to interest which becomes the connection mode of the network. High-quality products could be automatically delivered to consumers through this supply chain, which is a shortcut entrance.

\section{Communities based on social development of original e- commerce platform}

This pattern is mainly used in e-commerce platforms to improve users' interactivity. Simultaneously, they facilitate the information through the channels between social websites and e-commerce websites and collapse information island problem of traditional e-commerce websites in terms of information dissemination, such as Taojianghu in TaoBao.

1) The innate ability of gathering consumers.

All commercial cores are consumer relationships. TaoBao is born with the ability to gather consumers. Users come to TaoBao for consumption, thus TaoBao user relationship is formed by consumption demand. Other SNS shall be transformed through social relationships that are extracted from consumer relationships, but TaoBao has the inborn capability.

2) Users do not reject commerciality

If the direct relationship between utilitarian and commonweal could not be balanced appropriately in other SNS, the trust basis of communities will be shocked significantly. However, users come to TaoBao just for consumption, which creates a common demand and forms trust relationships. Therefore, TaoBao could absolutely gather social relationship via commercial demand.

3) Abundant user consumption behavior data

Users' behavior records mostly have nothing to do with consumption in other SNS, resulting in that their commercial value shall depend on commercial refined results of users' relationship and users' behaviors. It can be said that FACEBOOK is a diamond mine because there is a wealth of user social behavior data. However, if Facebook is a diamond mine, then TaoBao is the largest diamond in the mine with more than 400 million customers. TaoBao allows for clearer customer database processing of a customer's purchasing behavior which is invaluable to harnessing increased revenues.

\section{PROBLEMS ENCOUNTERED DURING THE} DEVELOPMENT PROCESS OF SOCIALIZATION E-COMMENCE

\section{A. Products recommended by acquaintances may not be what users are interested in}

Take Renren shopping as an example; products there are recommended by acquaintances to users. Users can see on their own pages the products their friends "are fond of," but they may not be interested in them. Acquaintances' recommendations could compress the information chain for users to find products at the maximum and users will trust recommended products more, but users only trust those products in which they share a common interest with their acquaintances; not everything their acquaintances share appeals to them.

1) Interested products may not come from acquaintances

It will be discovered that all users are interested in some product if one user clicks "shopping bar" or "recommendation" in Renren shopping, but the user is not familiar with most of them which results in that their fondness has nothing to do with the user. Therefore, acquaintances do not represent the overlap ratio of their purchased or interested products is high.

2) Consumers are not in a shopping mentally

Customer mentality is also "a gap" that needs to be bridged. People searching social websites like Renren are there to make friends, interact and play games instead of shopping. They are in a sharing mentality, not a shopping one.

\section{B. For shopping-guide communities based on interest connection}

\section{1) Insufficient user viscosity}

People search on these kinds of websites mostly for browsing. They will immediately move on if they find better shopping-guide websites. User viscosity is insufficient.

\section{2) Declinging Trust Factor}

The biggest problem caused by excessive participation on platforms, businesses and third-party providers is a trust decline, which is brought on by the conflict between community attributes and commercial attributes. Communities are originally socialization places for people to share information. However, businesses participate in communities mainly for profit. After they get involved overtly or covertly, information they issue must be undoubtedly favorable other than favorable or unfavorable evaluation issued by consumers based on real experience. Therefore, trust decline is inevitable.

\section{3) The value of shopping-guide communities is not} irreplaceable

Most shopping-guide communities in China currently are based on TaoBao. Actually, they only provide the value of the advertisement entrance, but could neither make users' behaviors become the closed loop nor form information systems. For example, modules like inner specials, groups, collocation, treasure show and professionals in Beauty. 
Discussion merely repeatedly provide advertisement entrance for TaoBao but do not solve the problem of users' behavior closed loop and information precipitation. Without the basis of TaoBao, their value will become extremely weak.

\section{For socialization platform with e-commerce as the core}

1) Enterprises'e-commerce property is difficult to transform to social property

The property of an enterprise is formed at the beginning of its establishment. For example, e-commerce platforms like TaoBao and Jingdong possess a massive shopping commercial property. A firm user relationship system similar to Renren could hardly be formed for the current situation of those enterprises and neither do an information flow system based on news information, entertainment information or gossip information and a diversified \& fragmented user relationship system like sina weibo. In this case, TaoBao turns around to operate social contact from a commercial state; its commercial property decides its impurity and the commercial property will affect users' intuitive judgment on the scope of business' and users' subconscious first choices.

2) E-commerce platforms values businesses' profit more

From the point of view of e-commerce, TaoBao attempts to pursue the balance between sellers and consumers, but "making easy business" indicates TaoBao is relatively biased toward sellers to some extent. Even though TaoBao claims to not be a company biased toward sellers, TaoBao could not be overwhelmingly biased toward consumers' interest and overwhelmingly stand for consumers.

3) Consumers' habitual thought could hardly change

From the point of view of consumers, this kind of platform is a place for businesses making a profit. The social function of this kind of platform will have a negative effect on consumer shopping, for any evaluation has the suspicion of marketing products and advertising. However, in shopping-guide communities, consumer perception is totally different for communities that are consumer-based and have fewer commercial advertisements.

\section{4) Shopping causes fragile relationships}

The relationship of TaoBao out of shopping is quite fragile. Most users are buying on their own initiative due to the high proportion of trading volume. Passive buying users are mostly from the outside. Besides, TaoBao users have little communication with each other.

\section{SUGGESTIONS FOR THE THREE MAIN DEVELOPMENT PATTERNS}

\section{A. For socialization e-commerce platform based on acquaintances}

1) Common interest between acquaintances must be taken into consideration

- Combine this with the Douban pattern and then screen out bosom friends with common interest in an acquaintance circle. For example, add a function of "recommending bosom friends" to help users find congenial friends, such as recommending friends of similar style or similar figure.
- Add the function of "labeling" after "like" button to classify and collect every interesting product by key words. Add labels users like in setting function of homepage to screen out products acquaintances "like." Thus, products that appear on the homepage will be orderly and what users and friends share a common interest in. This meets the individual style of users and launch products more accurately.

2) Shopkeepers are encouraged to set up shops in communities

Here are three forms: browsing shops (skip to business page to place orders and buy), direct selling shop (place orders and buy directly in community page) and fan shop (participating in interaction activities to achieve discount coupon, etc.).

3) Cooperation with large scale e-commerce

For example, cooperate with large scale e-commerce like TaoBao and Jingdong etc. Introduce friends to e-commerce to make their wish lists for birthdays and special events. In 2010, the successful cooperation between Facebook, Amazon and ebay provided a reference for similar cooperation in China.

\section{B. For shopping-guide communities}

\section{1) Rebuild consumer trust}

a) Solve reliability problem from UGC: UGC means “user generated content." UGC rose with Web2.0 mainly through features that are promoted individually. Main application forms of it are social network and various sharing communities. Unauthentic sources of UGC include conspirators, advertisements, spam and invalid comments. Establishing trust in UGC content in the Chinese online shopping environment is one of the keys of social sharing shopping-guide websites. For example, strictly distinguish sellers and buyers and maintain buyer angles and objectivity of sharing contents. Specifically, filtering unauthentic sources of contents could be done through modeling and summary of users' behaviors. The method of recognizing source reliability is based on that:

- Behaviors of suspicious users must be different from that of common users.

- Behavior pattern of long-term active users tend to be the same.

b) Obtain consumer trust by emotional shopping-guide: No longer make use of so-called socialization community function, but introduce some socialization deep applications further. For example, rebuild consumer trust and try to impress them mentally not physically in the form of subject shopping-guide and emotion shopping-guide. Those methods are mainly displayed in the context of a shoppingguide, i.e. set a more humane shopping-guide interface, introduce the product using different situations and weaken the description for products themselves in websites. For example, advertise products by some realistic themes and establish some life moment or life style to enable products to become a kind of taste or style conforming to this life situation or that life style, and thereby motivate consumers' 
purchasing desires. Purchase occurs here out of some emotional response from the consumers that is stimulated by the shopping-guide, such as a sense of identity and belonging. This kind of more humane shopping-guide website is worth investing in.

2) Enhance user viscosity

- Attract users by vouchers. Contacting users who are sharing products will obtain certain vouchers as long as transactions are concluded. Users could purchase any product in shopping-guide platform by vouchers, which is of great flexibility.

- If users are using the platform to share, they are more likely to continue buying from, and promoting, that platform.

C. For platforms with e-commerce as the core

- For the relationship produced by TaoBao out of shopping that is currently quite fragile, groups could be suggested to users based on their locations. For example, after buyers purchase chess from sellers on
TaoBao, they can recommend a local chess club, chess enthusiasts and chess activities to sellers.

- Subdivide contents. Since those people search for vertical social websites not for entertainment or making friends but for strong purpose, we could not regard such social websites as common comprehensive social websites, but put users' appeals first. Subdivide and label websites' contents to enable users to rapidly find them.

\section{REFERENCES}

[1] F.L. Fang, "Renren favorite-----Trust Impacts Purchase," Successful Marketing, No.4, 2012.pp.38

[2] Eguan, "Three Development Directions of Socialization Ecommerce,"

http://classroom.eguan.cn/moshi_143807.html,September 28, 2012.

[3] S. Jian, "Surpass FACEBOOK----Brief Talk of SNS Strategy," http://ec.sina.com.cn/ec/2011-08-18/16381.html, August 18, 2011.

[4] J. Fu, "Mertado.com: Socialization Shopping Platform," China $\begin{array}{lll}\text { Internet, } & \text { No.6, 2010.pp.77 }\end{array}$ 